\title{
Multiple complications of severe hyperandrogenism in a postmenopausal woman
}

\author{
Cristina Capatina*,** Alexandru Scafa-Udriste*, Adela Ghinea **, Anda Dumitrascu**, Catalina Poiana*, ${ }^{* *}$ \\ * "CAROL DAVILA" UNIVERSITY OF MEDICINE AND PHARMACY, BUCHAREST, ROMANIA \\ ** "C.I.PARHON » NATIONAL INSTITUTE OF ENDOCRINOLOGY AND METABOLISM, BUCHAREST, ROMANIA
}

\section{Introduction}

Severe hyperandrogenism in women is not only associated with cosmetic concerns but it also increases the risk of neoplastic (endometrial, breast cancer), cardiovascular, respiratory and metabolic complications.

\section{Case report}

A 68 years-old woman with a recent history of severe arterial hypertension, cardiogenic pulmonary edema, diabetes mellitus, severe respiratory failure, marked obesity was referred to our department due to her severely androgenic phenotype.

\section{Clinical examination}

The clinical examination showed android obesity, severe male-pattern/alopecia, marked hirsutism involving the face and the trunk, deepening of the voice.

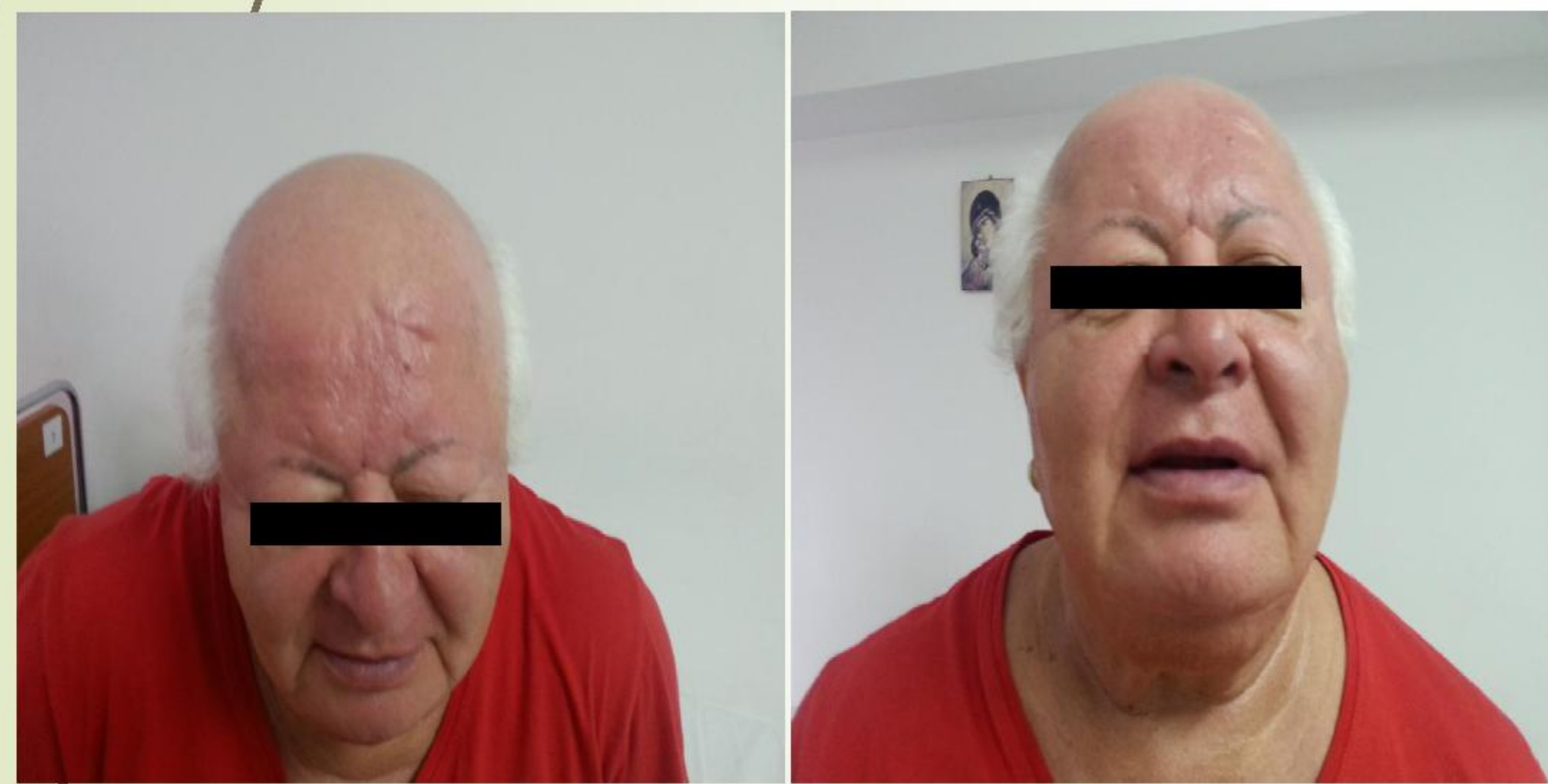

Severe, resistant arterial hypertension with hypertrophic cardiomyopathy and congestive heart failure were also resent. The patient associated chronic respiratory failure, severe obstructive sleep apnea syndrome, diabetes mellitus.

\section{Laboratory evaluation}

he endocrine evaluation revealed:

$\Rightarrow$ erythrocytosis

$\Rightarrow$ an extremely elevated serum testosterone level $>16.6 \mathrm{ng} / \mathrm{ml}$

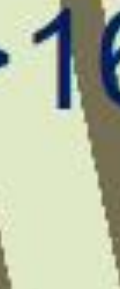

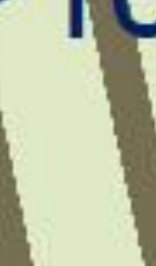

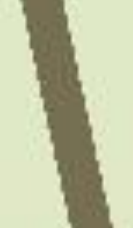

\section{Conclusions}

The presence of severe hyperandrogenism in postmenopausal women is frequently the result of an androgen-secreting tumor and can be associated with important complications.

Surgery was recommended and performed very recently, with significant early clinical improvement. As soon as 3 weeks after surgery the blood pressure and glycemic control improved markedly.

The histopathological examination revealed a benign ovarian thecoma with no suspicious endometrial findings. 